\title{
Characterizing the complex hyperbolic space by Kähler homogeneous structures *
}

\author{
P.M. Gadea, A. Montesinos Amilibia and J. Muñoz Masqué ${ }^{\dagger}$
}

\begin{abstract}
The characterization of the complex hyperbolic space equipped with the Bergman metric of negative constant holomorphic sectional curvature, as the unique (up to holomorphic isometries) simply connected irreducible homogeneous Kähler manifold admitting a nonvanishing Kähler homogeneous structure in Abbena-Garbiero's class $\mathcal{K}_{2} \oplus \mathcal{K}_{4}$, is given.
\end{abstract}

\section{Introduction}

The Kähler case of Riemannian homogeneous structures ([3, 15, 18]) has been studied in $[1,2,6,7,13,16]$, among other papers. Abbena and Garbiero [1] gave a classification of Kähler homogeneous structures, which has four primitive classes $\mathcal{K}_{1}, \ldots, \mathcal{K}_{4}$ (see [6, Th. 5.1] for another proof and $\S 2$ below for the result). The purpose of the present paper is to prove the following result:

Theorem 1.1. A simply connected irreducible homogeneous Kähler manifold admits a nonvanishing Kähler homogeneous structure in Abbena-Garbiero's class $\mathcal{K}_{2} \oplus \mathcal{K}_{4}$ if and only if it is the complex hyperbolic space equipped with the Bergman metric of negative constant holomorphic sectional curvature.

We thus have a situation similar to the Riemannian case, where a connected, simply connected and complete Riemannian manifold admits a nonvanishing homogeneous structure of first class if and only if it is isometric to the hyperbolic space ([18, Th. 5.2]), and similarly we obtain a vector field $\xi$ (see (4.4) in $§ 4$ ) which is the complex analog of the vector field in the Riemannian case satisfying $\nabla_{X} \xi=g(X, \xi) \xi-g(\xi, \xi) X([18,8])$, for Riemannian homogeneous structures of first class and models of negative constant (ordinary) sectional curvature. Moreover, this suggests the possibility of a quaternionic analog to Theorem 1.1 for models of negative constant quaternionic sectional curvature, and also of a Cayleyan analog.

*1991 AMS SCN: Primary 53C30; Secondary 53C25, 53C55. Key words: Kähler homogeneous structures, Kähler manifolds, Hermitian symmetric spaces, models of negative constant holomorphic sectional curvature.

${ }^{\dagger}$ Partially supported by the DGES (Spain): P.M.G and J.M.M. under Project PB-95-0124 and A.M.A. under Project PB-94-0972. 
On the other hand, similarly to the Riemannian case [18, p. 55], one has a solvable group acting simply transitively on the relevant domain (see Remark 4.2 , (i)), thus explaining why positive (holomorphic sectional) curvature is not detected.

\section{Preliminaries and notations}

As is well-known, Ambrose and Singer proved in [3] that a connected, simply connected and complete Riemannian manifold $(M, g)$ is homogeneous if and only if it admits a Riemannian homogeneous structure, i.e., a $(1,2)$ tensor field $S$ satisfying

$$
\widetilde{\nabla} g=0, \quad \widetilde{\nabla} R=0, \quad \widetilde{\nabla} S=0,
$$

where $\widetilde{\nabla}=\nabla-S, \nabla$ denotes the Levi-Civita connection and $R$ the curvature tensor of $\nabla$. We write $S_{X Y Z}=g\left(S_{X} Y, Z\right)$. Then, from $\nabla g=0$ it follows that the condition $\widetilde{\nabla} g=0$ is equivalent to $S_{X Z Y}=-S_{X Y Z}$. We set

$$
R_{X Y} Z=\nabla_{[X, Y]} Z-\nabla_{X} \nabla_{Y} Z+\nabla_{Y} \nabla_{X} Z, \quad R_{X Y Z W}=g\left(R_{X Y} Z, W\right),
$$

$R_{X Y}(Z, W)=R_{X Y Z W}, X, Y, Z, W \in \mathfrak{X}(M)$. In the sequel $X, Y, Z, W, U, \xi, \zeta$, will stand for vector fields on a $C^{\infty}$ manifold $M$. We denote by $F$ the Kähler form, defined by $F(X, Y)=g(X, J Y)$, by $r$ the Ricci tensor and by $s$ the scalar curvature.

Sekigawa proved ([17]) that a connected, simply connected and complete almost Hermitian manifold $(M, g, J)$ is homogeneous if and only if it admits an almost Hermitian homogeneous structure, i.e., a $(1,2)$ tensor field $S$ satisfying the conditions $(2.1)$ and $\widetilde{\nabla} J=0$.

We further suppose that $(M, g, J)$ is Kähler, so $\nabla J=0$. As $\widetilde{\nabla} J=0$, the condition $\nabla J=0$ is equivalent to $S_{X J Y J Z}=S_{X Y Z}$. Hence, if $S$ is an almost Hermitian homogeneous structure satisfying that invariance condition, then the manifold is Kähler ([1, Th. 5.8]). This property is equivalent to saying that $S$ belongs to the vector space

$$
\mathcal{S}(V)_{+}=\left\{S \in \otimes^{3} V^{*}: S_{X Y Z}=-S_{X Z Y}=S_{X J Y J Z}\right\} .
$$

Definition 2.1. A Kähler homogeneous structure on a Kähler manifold $(M, g$, $J)$ is an almost Hermitian homogeneous structure $S$ on $M$ such that $S_{x} \in$ $\mathcal{S}\left(T_{x} M\right)_{+}$for all $x \in M$.

The classification of Kähler homogeneous structures was obtained by Abbena and Garbiero in [1, Th. 2.1]. We recall here their result: Let $V$ be a $2 n$-dimensional real vector space (which is the model for the tangent space at any point of a manifold equipped with a Kähler homogeneous structure) endowed with a complex structure $J$ and a Hermitian inner product $\langle$,$\rangle , that$ is, $J^{2}=-I,\langle J X, J Y\rangle=\langle X, Y\rangle, X, Y \in V$, where $I$ denotes the identity isomorphism of $V$. 
If $\operatorname{dim} V \geq 6, \mathcal{S}(V)_{+}$decomposes into the direct sum of the following subspaces invariant and irreducible under the action of the group $U(n)$ :

$$
\begin{aligned}
& \mathcal{K}_{1}=\left\{S \in \mathcal{S}(V)_{+}: S_{X Y Z}=\frac{1}{2}\left(S_{Y Z X}+S_{Z X Y}\right.\right.+S_{J Y J Z X} \\
&\left.\left.+S_{J Z X J Y}\right), c_{12}(S)=0\right\} ; \\
& \mathcal{K}_{2}=\left\{S \in \mathcal{S}(V)_{+}: S_{X Y Z}=\langle X, Y\rangle \theta_{1}(Z)-\langle X, Z\rangle \theta_{1}(Y)+\langle X, J Y\rangle \theta_{1}(J Z)\right. \\
&\left.-\langle X, J Z\rangle \theta_{1}(J Y)-2\langle J Y, Z\rangle \theta_{1}(J X), \theta_{1} \in V^{*}\right\} ; \\
& \mathcal{K}_{3}=\left\{S \in \mathcal{S}(V)_{+}: S_{X Y Z}=-\frac{1}{2}\left(S_{Y Z X}+S_{Z X Y}+S_{J Y J Z X}\right.\right. \\
&\left.\left.\quad+S_{J Z X J Y}\right), c_{12}(S)=0\right\} ; \\
& \mathcal{K}_{4}=\left\{S \in \mathcal{S}(V)_{+}: S_{X Y Z}=\langle X, Y\rangle \theta_{2}(Z)-\langle X, Z\rangle \theta_{2}(Y)+\langle X, J Y\rangle \theta_{2}(J Z)\right. \\
&\left.-\langle X, J Z\rangle \theta_{2}(J Y)+2\langle J Y, Z\rangle \theta_{2}(J X), \theta_{2} \in V^{*}\right\} ;
\end{aligned}
$$

$X, Y, Z \in V$, where $c_{12}$ is defined by $c_{12}(S)(X)=\sum_{i=1}^{2 n} S_{e_{i} e_{i} X}$, for any $X \in V$; $\left\{e_{1}, \ldots, e_{2 n}\right\}$ denotes an arbitrary orthonormal basis of $V$; and

$$
\theta_{1}(X)=\frac{1}{2(n-1)} c_{12}(S)(X), \quad \theta_{2}(X)=\frac{1}{2(n+1)} c_{12}(S)(X), \quad X \in V .
$$

If $\operatorname{dim} V=4$, then $\mathcal{S}(V)_{+}=\mathcal{K}_{2} \oplus \mathcal{K}_{3} \oplus \mathcal{K}_{4}$. If $\operatorname{dim} V=2$, then $\mathcal{S}(V)_{+}=\mathcal{K}_{4}$.

\section{The class $\mathcal{K}_{2} \oplus \mathcal{K}_{4}$}

We can write the class $\mathcal{K}_{2} \oplus \mathcal{K}_{4}$ as

$$
\begin{aligned}
\mathcal{K}_{2} \oplus \mathcal{K}_{4}=\{S & \in \mathcal{S}(V)_{+}: S_{X Y Z}=\langle X, Y\rangle\left(\theta_{1}+\theta_{2}\right)(Z) \\
& -\langle X, Z\rangle\left(\theta_{1}+\theta_{2}\right)(Y)+\langle X, J Y\rangle\left(\theta_{1}+\theta_{2}\right)(J Z) \\
& \left.-\langle X, J Z\rangle\left(\theta_{1}+\theta_{2}\right)(J Y)+2\langle Y, J Z\rangle\left(\theta_{1}-\theta_{2}\right)(J X)\right\} .
\end{aligned}
$$

Remark 3.1. We recall that $\mathcal{K}_{2} \oplus \mathcal{K}_{4}$ is the sum of the isotypic components $\mathcal{K}_{2}$, $\mathcal{K}_{4}$ of the smallest dimension in the decomposition of $\mathcal{S}(V)_{+}$above. In fact, one has $\mathcal{K}_{2} \cong \mathcal{K}_{4} \cong V \cong V^{*}$, and the respective dimensions of $\mathcal{K}_{1}, \ldots, \mathcal{K}_{4}$ are (see [1, p. 382]) $n(n+1)(n-2), 2 n, n(n-1)(n+2), 2 n$. It is thus reasonable that $\mathcal{K}_{2} \oplus \mathcal{K}_{4}$ corresponds to spaces of (negative) constant holomorphic sectional curvature, which are scarce in all homogeneous Kähler spaces.

Lemma 3.2. A simply connected complete irreducible Kähler manifold $(M=$ $G / H, g, J)$ of real dimension $2 n \geq 4$ which admits a nonvanishing Kähler homogeneous structure $S \in \mathcal{K}_{2} \oplus \mathcal{K}_{4}$, is Einstein.

Proof. Let $\xi$ be the vector field dual to the 1-form $\theta=\theta_{1}+\theta_{2}$ and $\zeta$ the vector field dual to the 1 -form $\theta_{1}-\theta_{2}$, both with respect to the metric. From (3.1) we have

(3.2) $S_{X} Y=g(X, Y) \xi-g(Y, \xi) X-g(X, J Y) J \xi+g(J Y, \xi) J X-2 g(J X, \zeta) J Y$. 
Taking the $\widetilde{\nabla}_{Z}$ derivative of this formula, applying Ambrose-Singer's equations (2.1) and Sekigawa's equation $\widetilde{\nabla} J=0$, and contracting with $g(W, \cdot)$, we obtain

$$
\begin{aligned}
g(X, Y) g\left(\widetilde{\nabla}_{Z} \xi, W\right) & -g\left(\widetilde{\nabla}_{Z} \xi, Y\right) g(X, W)-g(X, J Y) g\left(\widetilde{\nabla}_{Z} J \xi, W\right) \\
& +g\left(\widetilde{\nabla}_{Z} \xi, J Y\right) g(J X, W)-2 g\left(J X, \widetilde{\nabla}_{Z} \zeta\right) g(J Y, W)=0 .
\end{aligned}
$$

Since $\operatorname{dim} M \geq 4$, we deduce

$$
\widetilde{\nabla} \xi=0, \quad \widetilde{\nabla} \zeta=0 .
$$

Thus, from $\widetilde{\nabla} g=0$ we have $g(\xi, \xi)=$ const, $g(\zeta, \zeta)=$ const. If $\xi=0, \zeta \neq 0$, we also have

$$
R_{X Y} \zeta=4(g(X, J \zeta) g(Y, \zeta)-g(X, \zeta) g(Y, J \zeta)) J \zeta .
$$

The second Ambrose-Singer condition in (2.1) can be written as

$$
\left(\nabla_{X} R\right)_{Y Z W U}=-R_{S_{X} Y Z W U}-R_{Y S_{X} Z W U}-R_{Y Z S_{X} W U}-R_{Y Z W S_{X} U} .
$$

Suppose $\xi \neq 0$. Applying Bianchi's second identity to (3.5) and then substituting (3.2), we obtain

$$
\begin{aligned}
& \mathfrak{S}_{X Y Z}\left\{2 g(X, \xi) R_{Y Z W U}+g(X, W) R_{Y Z \xi U}+g(X, U) R_{Y Z W \xi}\right. \\
& \left.-2 g(X, J Y) R_{J \xi Z W U}-g(X, J W) R_{Y Z J \xi U}-g(X, J U) R_{Y Z W J \xi}\right\}=0 .
\end{aligned}
$$

Contracting (3.6) with respect to $X$ and $W$ and applying Bianchi's first identity, we deduce

$$
\begin{aligned}
& (2 n+2) R_{Z Y \xi U}=-2 g(Y, \xi) r(Z, U)+2 g(Z, \xi) r(Y, U) \\
& +2 g(Y, J Z) r(J \xi, U)-g(Y, U) r(Z, \xi)+g(Y, J U) r(Z, J \xi) \\
& +g(Z, U) r(Y, \xi)-g(Z, J U) r(Y, J \xi) .
\end{aligned}
$$

Contracting (3.7) with regard to $Y$ and $U$, we obtain $r(Z, \xi)=(s / 2 n) g(Z, \xi)$. Putting $a=1 /(2 n+2), b=s / 2 n$, we can write (3.7) as

$$
\frac{1}{a} R_{\xi U}=2 \theta \wedge r(U)+2 b \theta(J U) F+b U^{b} \wedge \theta+b(J U)^{b} \wedge(\theta \circ J) .
$$

On the other hand, from Bianchi's first identity one has $R_{W U}(J \xi, \cdot)=$ $R_{\xi J W}(U, \cdot)-R_{\xi J U}(W, \cdot)$. Thus we can write $(3.6)$ as

$$
\begin{aligned}
2 \theta \wedge R_{W U}+W^{\mathrm{b}} \wedge R_{\xi U}-U^{\mathrm{b}} \wedge & R_{\xi W}+2 F \wedge\left(R_{\xi J U}(W, \cdot)-R_{\xi J W}(U, \cdot)\right) \\
& +(J W)^{\mathrm{b}} \wedge R_{\xi J U}-(J U)^{\mathrm{b}} \wedge R_{\xi J W}=0
\end{aligned}
$$


Substituting (3.8) in (3.9) and denoting by $\Xi(U)$ the right hand side of (3.8), we deduce

$$
\begin{aligned}
\frac{2}{a} \theta \wedge R_{W U} & +W^{\mathrm{b}} \wedge \Xi(U)-U^{\mathrm{b}} \wedge \Xi(W)+2 F \wedge\left(i_{W}(\Xi(J U))\right. \\
& \left.-i_{U}(\Xi(J W))\right)+(J W)^{\mathrm{b}} \wedge \Xi(J U)-(J U)^{\mathrm{b}} \wedge \Xi(J W)=0 .
\end{aligned}
$$

Taking $W=\xi$ in this formula we obtain

$$
(2 g(\xi, \xi) F+\theta \wedge(\theta \circ J)) \wedge\left(r(J U)-b(J U)^{b}\right)=0 .
$$

Contracting this formula with $\xi$, in account of $r(\xi, \cdot)=b g(\xi, \cdot)$ we deduce

$$
g(\xi, \xi)(\theta \circ J) \wedge\left(r(J U)-b(J U)^{b}\right)=0 .
$$

Contracting with $J \xi$ we have $g(\xi, \xi)^{2}\left(r(J U)-b(J U)^{b}\right)=0$. Since $\xi \neq 0$ we conclude that $r=b g$, that is, $(M, g, J)$ is Einstein.

Now suppose $\xi=0, \zeta \neq 0$. It is immediate to see that $[\zeta, J \zeta]=-2 g(\zeta, \zeta) J \zeta$, so that $M$ has a 2-dimensional involutive distribution, which is also parallel as follows from (3.2) and $\widetilde{\nabla} \zeta=0$ in (3.3). Thus ([5, Prop. 10.21]) the holonomy representation $\operatorname{Hol}(g)$ leaves invariant a subspace of dimension 2. So, by the De Rham theorem ([5, Th. 10.41]), we conclude that $M$ is holomorphically isometric to a Kähler product, but then $M$ would not be irreducible.

Theorem 3.3. A simply connected irreducible homogeneous Kähler manifold of real dimension $2 n \geq 4$ admitting a nonvanishing Kähler homogeneous structure $S \in \mathcal{K}_{2} \oplus \mathcal{K}_{4}$ is holomorphically isometric to a bounded symmetric domain of negative constant holomorphic sectional curvature.

Proof. Suppose $\xi \neq 0$. Since by the Lemma the manifold is Einstein, (3.10) gives us

$$
\theta \wedge\left(\frac{1}{a b} R_{W U}+U^{b} \wedge W^{b}+(J U)^{b} \wedge(J W)^{b}-2 F(W, U) F\right)=0 .
$$

Contracting this formula with $\xi$ we deduce, being $k=g(\xi, \xi)$,

$$
\begin{aligned}
& \frac{k}{a b} R_{W U}-k\left(W^{\mathrm{b}} \wedge U^{\mathrm{b}}+(J W)^{\mathrm{b}} \wedge(J U)^{\mathrm{b}}+2 F(W, U) F\right)=\theta \wedge\left(\frac{1}{a b} R_{W U}(\xi, \cdot)\right. \\
& \left.+\theta(U) W^{\mathrm{b}}-\theta(W) U^{\mathrm{b}}+\theta(J U)(J W)^{\mathrm{b}}-\theta(J W)(J U)^{\mathrm{b}}-2 F(W, U)(\theta \circ J)\right) .
\end{aligned}
$$

On the other hand, from Bianchi's first identity we deduce $R_{W U}(\xi, \cdot)=$ $R_{\xi U}(W, \cdot)-R_{\xi W}(U, \cdot)$. Substituting these two summands by their expression from (3.8) and then substituting the expression for $R_{W U}(\xi, \cdot)$ in (3.12) we obtain

$$
\frac{1}{a b} R_{W U}=W^{b} \wedge U^{b}+(J W)^{b} \wedge(J U)^{b}+2 F(W, U) F,
$$


from which

$$
\begin{array}{r}
R_{Y Z W U}=\frac{s}{4 n(n+1)}\{g(Y, W) g(Z, U)-g(Y, U) g(Z, W)+g(Y, J W) g(Z, J U) \\
-g(Y, J U) g(Z, J W)+2 g(Y, J Z) g(W, J U)\} .
\end{array}
$$

That is, $(M, g, J)$ is a model of constant holomorphic sectional curvature $c=$ $s / n(n+1)$. To determine the sign of $s$, we prove that (being $\xi=0$ or not) if $(M, g, J)$ is a space of constant holomorphic sectional curvature $c$, then $\zeta=0$ and $c=-g(\xi, \xi)$. In fact, comparing the expressions for $R_{X J X} \xi$ respectively obtained from substitution in the usual expression

$R_{X Y} Z=c\{g(X, Z) Y-g(Y, Z) X+g(J X, Z), J Y-g(J Y, Z) J X+2 g(X, J Y) J Z\}$, and from $\nabla_{X} \xi=S_{X} \xi$, since $n \geq 2$ we obtain the system of equations

$$
\begin{aligned}
& g(X, \xi) g(J X, \zeta)-g(J X, \xi) g(X, \zeta)=0 \\
& c g(X, \xi)=-g(\xi, \xi) g(X, \xi-\zeta) \\
& 0=(2 g(\xi, \zeta)-g(\xi, \xi)-c) g(X, X)+g(X, \xi) g(X, \zeta) \\
& \quad+g(J X, \xi) g(J X, \zeta)-2 g(X, \zeta)^{2}-2 g(J X, \zeta)^{2}
\end{aligned}
$$

From (3.14) we have $g(\xi, \xi) \zeta=(g(\xi, \xi)+c) \xi$ that, if is true, implies (3.13). In (3.15), we can take $X$ orthogonal to $\zeta$ and $J \zeta$, so $g(\xi, \xi)+c=2 g(\xi, \zeta)$, thus $g(\xi, \xi)(g(\xi, \xi)+c)=2 g(\xi, g(\xi, \xi) \zeta)=2 g(\xi, \xi)(g(\xi, \xi)+c)$, hence

$$
g(\xi, \xi)(g(\xi, \xi)+c)=0
$$

Suppose $g(\xi, \xi)=0$. As $\xi=0,(3.15)$ simplifies to

$$
c g(X, X)+2 g(X, \zeta)^{2}+2 g(J X, \zeta)^{2}=0 .
$$

Since $\operatorname{dim} M \geq 4$, we can take $X$ orthogonal to $\zeta$ and to $J \zeta$, so $c g(X, X)=0$, that is, the manifold is flat. From (3.4) we deduce

$$
g(X, \zeta) g(J Y, \zeta)-g(Y, \zeta) g(J X, \zeta)=0
$$

Taking $X=\zeta$ we have $g(\zeta, \zeta) \zeta=0$, which implies $\zeta=0$, but then $S$ vanishes.

On the other hand, if $g(\xi, \xi) \neq 0$, then (3.16) gives $g(\xi, \xi)+c=0$; hence $\zeta=0$.

The case $\xi=0, \zeta \neq 0$ has been already discarded in the previous Lemma.

Remark 3.4. In the conditions of Lemma 3.2, for $\xi=0, \zeta \neq 0$, substituting (3.2) in (3.5), as $\nabla J=0$ we have

$$
\left(\nabla_{X} R\right)_{Y Z W U}=2 g(J X, \zeta)\left(R_{J Y Z W U}+R_{Y J Z W U}+R_{Y Z J W U}+R_{Y Z W J U}\right)=0 .
$$

Thus $(M, g, J)$ is Hermitian symmetric. As we have seen, it is holomorphically isometric to a Kähler product, with a 2-dimensional factor, which is a space of negative constant curvature $-4 g(\zeta, \zeta)$, as it follows from (3.4). 
Now, there are ([10, pp. 518-20]) four nonflat noncompact Hermitian symmetric surfaces: $S U(1,1) / S(U(1) \times U(1)), S O^{*}(4) / U(2), S O_{0}(2,1) / S O(2) \times 1$ and $S p(1) / U(1)$. All of them are isomorphic, and can be identified to the real hyperbolic space $S O_{0}(2,1) / S O(2) \times 1$. Taking the half-plane Poincaré model $H=\{z=x+i y \in \mathbb{C}: y>0\}$ for it, we have the metric and the complex structure (particular cases of (4.2) and (4.3) below)

$$
g=-\frac{\mathrm{d} x^{2}+\mathrm{d} y^{2}}{c y^{2}}, \quad J=\frac{\partial}{\partial y} \otimes \mathrm{d} x-\frac{\partial}{\partial x} \otimes \mathrm{d} y .
$$

It is immediate that the vector field $\zeta=-(c y / 2) \partial / \partial y$ (a particular case of $(4.4)$ below) satisfies the equation $\nabla_{X} \zeta=2 g(X, J \zeta) J \zeta, \nabla$ being the Levi-Civita connection of $g$. We conclude that in the simply connected reducible case one can also consider spaces holomorphically isometric to products of the 2-dimensional Siegel domain model of the complex hyperbolic space, that is, the above halfplane Poincaré model, equipped with the Kähler homogeneous structure $S$ given by $S_{X} Y=2 g(X, J \zeta) J Y$, and any other Hermitian symmetric space, endowed with its standard vanishing Kähler homogeneous structure as a Hermitian symmetric space.

\section{Proof of Theorem 1.1}

As for the converse, it suffices to give a nonvanishing Kähler homogeneous structure on any model of negative constant holomorphic sectional curvature. Thus, to finish the proof we explicitly give such a structure on the Siegel domain

$$
D_{+}=\left\{\left(z=x+i y, u^{1}, \ldots, u^{n}\right) \in \mathbb{C}^{n+1}: y-\sum_{k=1}^{n} u^{k} \bar{u}^{k}>0\right\},
$$

equipped with the Kähler structure obtained by a convenient Cayley transform ([14, p. 5]) from that of the unit open ball in $\mathbb{C}^{n+1}([9$, p. 227] or $[12$, p. 169], changing the sign in the second summand of the numerator in the last expression of the metric). A calculation shows that

$$
\begin{aligned}
g_{+}=-\frac{1}{c\left(y-\sum u^{k} \bar{u}^{k}\right)^{2}}\left\{\mathrm{~d} z \mathrm{~d} \bar{z}+4\left(y-\sum u^{k} \bar{u}^{k}\right) \sum \mathrm{d} u^{k} \mathrm{~d} \bar{u}^{k}\right. \\
\left.+2 i\left(\mathrm{~d} z \sum u^{k} \mathrm{~d} \bar{u}^{k}-\mathrm{d} \bar{z} \sum \bar{u}^{k} \mathrm{~d} u^{k}\right)+4\left(\sum \bar{u}^{k} \mathrm{~d} u^{k}\right)\left(\sum u^{k} \mathrm{~d} \bar{u}^{k}\right)\right\} .
\end{aligned}
$$

$\left(D_{+}, g_{+}, J_{+}\right)$is a Kähler manifold, with

$$
J_{+}=i\left(\frac{\partial}{\partial z} \otimes \mathrm{d} z+\sum_{k=1}^{n} \frac{\partial}{\partial u^{k}} \otimes \mathrm{d} u^{k}-\frac{\partial}{\partial \bar{z}} \otimes \mathrm{d} \bar{z}-\sum_{k=1}^{n} \frac{\partial}{\partial \bar{u}^{k}} \otimes \mathrm{d} \bar{u}^{k}\right) .
$$

The Riemannian manifold $\left(D_{+}, g_{+}\right)$is homogeneous, hence complete. Since $\left(D_{+}, g_{+}, J_{+}\right)$is connected, simply connected and complete, it is a model of 
negative constant holomorphic sectional curvature. Furthermore, we seek for a homogeneous structure $S$ of the type

$$
S_{X} Y=g(X, Y) \xi-g(\xi, Y) X-g(X, J Y) J \xi+g(\xi, J Y) J X .
$$

Since $\widetilde{\nabla} \xi=0$, we must find a vector field $\xi$ on $D_{+}$satisfying

$$
\nabla_{X} \xi=g(X, \xi) \xi-g(\xi, \xi) X-g(X, J \xi) J \xi
$$

Putting $u^{k}=v^{k}+i w^{k}$, the metric $g_{+}$and the complex structure $J_{+}$are written as

$$
\begin{aligned}
& \text { (4.2) } g_{+}=-\frac{1}{c\left[y-\sum\left(\left(v^{k}\right)^{2}+\left(w^{k}\right)^{2}\right)\right]^{2}}\left\{\mathrm{~d} x^{2}+\mathrm{d} y^{2}+4\left[y-\sum_{j \neq k}\left(\left(v^{j}\right)^{2}+\left(w^{j}\right)^{2}\right)\right]\right. \\
& \cdot \sum_{k}\left(\left(\mathrm{~d} v^{k}\right)^{2}+\left(\mathrm{d} w^{k}\right)^{2}\right)-4\left[\mathrm{~d} x \sum\left(w^{k} \mathrm{~d} v^{k}-v^{k} \mathrm{~d} w^{k}\right)-\mathrm{d} y \sum\left(v^{k} \mathrm{~d} v^{k}+w^{k} \mathrm{~d} w^{k}\right)\right] \\
& \left.+8 \sum_{k, l}\left[\left(v^{k} v^{l}+w^{k} w^{l}\right)\left(\mathrm{d} v^{k} \mathrm{~d} v^{l}+\mathrm{d} w^{k} \mathrm{~d} w^{l}\right)+\left(v^{l} w^{k}-v^{k} w^{l}\right)\left(\mathrm{d} v^{k} \mathrm{~d} w^{l}-\mathrm{d} w^{k} \mathrm{~d} v^{l}\right)\right]\right\}
\end{aligned}
$$

$$
J_{+}=\frac{\partial}{\partial y} \otimes \mathrm{d} x-\frac{\partial}{\partial x} \otimes \mathrm{d} y+\sum\left(\frac{\partial}{\partial w^{k}} \otimes \mathrm{d} v^{k}-\frac{\partial}{\partial v^{k}} \otimes \mathrm{d} w^{k}\right)
$$

A long but straightforward computation shows that the vector field

$$
\xi=-\frac{c}{2}\left(y-\sum u^{k} \bar{u}^{k}\right) \frac{\partial}{\partial y}
$$

satisfies (4.1) for all $X$. Specifically, if $X$ is given by

$$
X=\alpha \frac{\partial}{\partial x}+\beta \frac{\partial}{\partial y}+\sum\left(\gamma_{k} \frac{\partial}{\partial v^{k}}+\delta_{k} \frac{\partial}{\partial w^{k}}\right)
$$

we obtain

$$
\begin{aligned}
\nabla_{X} \xi=\frac{c}{4}\left\{2 \left[\left(\alpha+\sum\left(\delta_{k} v^{k}-\gamma_{k} w^{k}\right)\right) \frac{\partial}{\partial x}+\sum\right.\right. & \left.\left(\gamma_{k} v^{k}+\delta_{k} w^{k}\right) \frac{\partial}{\partial y}\right] \\
& \left.+\sum\left(\gamma_{k} \frac{\partial}{\partial v^{k}}+\delta_{k} \frac{\partial}{\partial w^{k}}\right)\right\} .
\end{aligned}
$$

Hence, $\left(D_{+}, g_{+}, J_{+}\right)$admits the Kähler homogeneous structure $S$ given by

$$
\begin{aligned}
S_{X} Y=-\frac{c}{2}\left(y-\sum u^{k} \bar{u}^{k}\right)\{ & g(X, Y) \frac{\partial}{\partial y}-g\left(\frac{\partial}{\partial y}, Y\right) X \\
& \left.+g(X, J Y) \frac{\partial}{\partial x}+g\left(\frac{\partial}{\partial y}, J Y\right) J X\right\} .
\end{aligned}
$$

According to Heintze's Theorem ([11, Th. 4]), a connected homogeneous Kähler manifold of negative curvature is holomorphically isometric to the complex hyperbolic space. Hence 
Corollary 4.1. A connected homogeneous Kähler manifold of real dimension $2 n \geq 4$ and negative curvature admits a nonvanishing Kähler homogeneous structure $S \in \mathcal{K}_{2} \oplus \mathcal{K}_{4}$.

Remark 4.2. (i) The Siegel domain $D_{+}$thus admits at least two Kähler homogeneous structures: $S=0$, as $D_{+}$is a realization of the noncompact Hermitian symmetric space $U(1, n) / U(1) \times U(n)$; and the nonvanishing structure $S$ in (4.5), corresponding to the fact that the solvable group $\mathbb{C} H^{n}$ (see [11, p. 33]) acts simply transitively on $D_{+}$(see $[11$, p. 32], [5, p. 181], [4, p. 92]) by holomorphic isometries. Hence, we can identify $D_{+}$with $\mathbb{C} H^{n}$ and thus it is immediate to obtain the expression of the canonical connection on the reductive homogeneous space $\left(D_{+}, g_{+}, J_{+}\right)$identified to the solvable group $\mathbb{C} H^{n}$.

(ii) For $\operatorname{dim} M=2$ it only rests the class $\mathcal{K}_{4}$. This case has been studied by Abbena and Garbiero [1, p. 391].

\section{References}

[1] E. Abbena and S. Garbiero, Almost Hermitian homogeneous structures, Proc. Edinb. Math. Soc. (2) 31 (1988), 375-395.

[2] E. Abbena and S. Garbiero, Almost Hermitian homogeneous manifolds and Lie groups, Nihonkai Math. J. 4 (1993), 1-15.

[3] W. Ambrose and I.M. Singer, On homogeneous Riemannian manifolds, Duke Math. J. 25 (1958), 647-669.

[4] J. Berdnt, F. Tricerri, and L. Vanhecke, Generalized Heisenberg groups and Damek-Ricci harmonic spaces (Springer-Verlag, 1995).

[5] A. BEsse, Einstein manifolds (Springer-Verlag, 1987).

[6] S. Console and A. Fino, Homogeneous structures on Kähler submanifolds of complex projective spaces, Proc. Edinb. Math. Soc. (2) 39 (1996), 381-395.

[7] M. Falcitelli, A. Farinola and S. Salamon, Almost-Hermitian geometry, Diff. Geom. Appl. 4 (1994), 259-282.

[8] P.M. Gadea and J.A. OubiÑA, Reductive homogeneous pseudo-Riemannian manifolds, Monatsh. Math. 124 (1997), 17-34.

[9] S. GoldBerg, Curvature and homology (Dover, 1982).

[10] S. Helgason, Differential geometry, Lie groups and symmetric spaces (Academic Press, 1978).

[11] E. Heintze, On homogeneous manifolds of negative curvature, Math. Ann. 211 (1974), 23-34.

[12] S. Kobayashi and K. Nomizu, Foundations of Differential Geometry, II (Intersc. Publ., 1969).

[13] A. Ledger and L. Vanhecke, Naturally reductive s-manifolds, Note Mat. 10 (1990), 363-370.

[14] S. Murakami, Automorphisms of Siegel domains, Lect. Notes in Math. vol. 286 (Springer-Verlag, 1972). 
[15] V. Patrangenaru, Classifying 3- and 4-dimensional homogeneous Riemannian manifolds by Cartan triples, Pacific J. Math. 173 (1996), 511-532.

[16] S. Salamon, Riemannian geometry and holonomy groups (Longman, Essex, 1989).

[17] K. Sekigawa, Notes on homogeneous almost Hermitian manifolds, Hokkaido Math. J. 7 (1978), 206-213.

[18] F. Tricerri and L. VANHeCKe, Homogeneous structures on Riemannian manifolds, London Math. Soc. Lect. Notes Ser. vol. 83 (Cambridge Univ. Press, 1983).

[19] J.A. Wolf, Spaces of constant curvature (Publish or Perish, 1977).

Authors' addresses:

P.M.G.: Institute of Mathematics and Fundamental Physics, CSIC, Serrano 123, 28006-Madrid, Spain, e-mail: pmgadea@gugu.usal.es

A.M.A.: Department of Geometry and Topology, Faculty of Mathematics, 46100Burjasot, Valencia, Spain, e-mail: Angel.Montesinos@uv.es

J.M.M.: Institute of Applied Physics, CSIC, Serrano 144, 28006-Madrid, Spain, email: jaime@iec.csic.es 